\title{
Identidad de género: desafíos y límites a las políticas de inclusión en la escuela secundaria de la Ciudad de Buenos Aires, Argentina
}

Gender identity: challenges and limits to the inclusion policies in high school in Buenos Aires city, Argentina.

Jesica Baez*

\section{Resumen}

Este artículo tiene por objeto explorar las formas de transitar la escuela secundaria (gestión pública) de jóvenes trans que se reconocen como travestis, transexuales, transgéneros, feminidades o masculinidades trans en la Ciudad de Buenos Aires, Argentina. Para ello he construido un corpus empírico constituido por la reconstrucción del caso de un estudiante, una serie de entrevistas informales a docentes y estudiantes, observaciones de trabajo de campo en escuela públicas secundarias de la Ciudad de Buenos de Aires entre el 2009 y el 2012, documentos y finalmente, una serie de estadísticas elaboradas por organizaciones sociales que luchan por los derechos de los personas trans y los resultados de la prueba piloto de la Primera Encuesta Nacional sobre la Población Trans: Travestis, Transexuales, Transgéneros y Hombres realizada por el INDEC - INADI durante 2012.

Palabras claves: Identidad de género - Educación secundaria - Políticas públicas Educación sexual - Jóvenes.

\begin{abstract}
This article aims to explore the educational experience at High School (Public) trans youth are recognized as transvestites, transsexuals, transgender or trans masculinity or femininity in the Buenos Aires City, Argentina. For it built this empirical corpus constituted by reconstruction case of a student, a series of informal interviews students and teachers, observations of field work in High Public School of Buenos Aires between 2009 and 2012, documents, and finally a series of statistics compiled by social organizations that fight for the rights of trans characters and the results of the pilot of the First National Population Survey trans test: Transvestites, Transsexuals and Transgender INDEC - INADI (2012).
\end{abstract}

Keywords: Gender identity - High School - Public Policy - Sexual Education - Youth

Fecha de recepción: 31 de agosto 2015

\footnotetext{
* Dra. en Educación de la Facultad de Filosofía y Letras de la Universidad de Buenos Aires, Jefa de Trabajos Prácticos de la asignatura Investigación y estadística educacional II y Profesora del Seminario Abordaje socioeducativo de las problemáticas de género y sexualidades de FFYL, UBA. Becaria posdoctoral del CONICET.
} 
Fecha de aprobación: 13 de marzo 2017

Una tarde, nos encontramos con la asesora en su oficina a conversar. Un tanto retrasada, llega caminando por el pasillo, al acercarse me dice: Asesora: iNo sabes lo que me paso! iMe quiero morir!

Observadora: ¿Qué?

Asesora: Entre a un curso de Primer año, no tenían profesor. Entre para preguntarles en qué andaban. Había algunos chicos parados, entonces me acerco a una chica, y le digo al resto: “Ven, ¿ por qué no se portan como ella?". Estaban haciendo ruido, y ella estaba ahí

tranquila.

Observadora: Claro.

Asesora: Miro a los estudiantes, y veo que algunos se ríen, y otros me miran.

Observadora: ¿Y qué paso?

Asesora: Yo seguí, diciéndoles que se porten bien y me fui. Y ahí me di cuenta. No era una chica, era un varón. Tenía los ojos, así delineados y no se molestó, me pareció cuando le

dije. Sí, en cambio, el grupo se quedó así (abre sus ojos).

Con el tiempo, comencé a preguntar por ese/a chico/a, particularmente a los/as adultos/as. Una serie de frases comenzaron a repetirse:

"hoy no vino"

"¿acá? ¿Seguro?"

"no hay, no hay chicos asi"

(Conversación informal en el patio de una escuela secundaria pública secundaria, Junio de

\section{Viñeta 1}

\section{Sobre las delimitaciones imprecisas: jóvenes trans en la escuela secundaria}

Este artículo tiene por objeto explorar las formas de transitar la escuela secundaria de jóvenes trans que se reconocen como feminidades o masculinidades trans ${ }^{1}$. La viñeta 1 condensa una serie de sentidos que desplegaré en el desarrollo de los próximos párrafos y que provienen de una escena acontecida durante mi trabajo de tesis doctoral ${ }^{2}$ : ¿Qué sucede en esta escena inicial? Junto con otros diálogos "entrecortados" y "huidizos" que

\footnotetext{
${ }^{1}$ En el desarrollo del trabajo de investigación se ha respetado las formas de nominación que los/as/xs jóvenes se asignaban en relación a su sexo/género. Utilizó en este escrito el término feminidades/masculinidades trans como vocablo que agrupa las múltiples maneras de reconocimiento de los/as/xs estudiantes. Algunos de ellos/as/xs se denominaban travestis, otros/as como raros/as. En este artículo se recupera el nombre propio que cada unos $/ \mathrm{a} / \mathrm{x}$ decidió para ser publicado a tales fines.

${ }_{2}^{2}$ Tesis doctoral titulada: La experiencia educativa trans. Los modos de vivir el cuerpo sexuado de los/as/xs jóvenes en la escuela secundaria de la Ciudad de Buenos Aires, dirigida por la Dra. Graciela Morgade y codirigida por la Dra. Ana Padawer en el marco de la Facultad de Filosfoía y Letras, Universidad de Buenos Aires y con el apoyo de la beca doctoral CONICET. Defendida en el mes de marzo de 2014.
} 
han sucedido en mi paso por una escuela pública secundaria, y también en el tránsito por otras escuelas secundarias comunes públicas de la Ciudad de Buenos Aires.

En el trabajo con estas instituciones llegaron a mis oídos historias como la de la viñeta, historias "de chicos" que dejaban la escuela y que con el correr del tiempo alguien los cruzaba por la calle y estaban vestidos de "mujer". Estos relatos dan cuenta de estudiantes que desde los ojos de los otros son identificadas como "travestis". Otros relatos, no obstante, aparecen totalmente invisibilizados: por ejemplo una estudiante mujer que transita la escuela reconociéndose como varón.

Estas imágenes brindan algunas pistas de lo complejo de abordar el tema en su conjunto y al interior del mismo. Por un lado, porque las categorías "travesti", "transexual" y "transgénero" han sido objeto de múltiples luchas por sus sentidos. El discurso médicopsiquiátrico, el engranaje judicial-policial, los usos en los medios de comunicación, los movimientos de lucha socio-sexual, por ejemplo, han sido participes de tal proceso. Por el otro, porque indagar la experiencia de estos/as/xs jóvenes en la escuela común, también, es analizar el por qué son excluidos de ella.

En síntesis, en este artículo propongo indagar la experiencia educativa de estudiantes travestis, transgéneros y transexuales o bien, que se reconocen como feminidades o masculinidades trans en relación a la escuela secundaria. Para ello construí un corpus empírico constituido por la reconstrucción del caso de un estudiante, una serie de entrevistas informales a docentes y estudiantes, observaciones de trabajo de campo en escuela públicas secundarias de la Ciudad de Buenos de Aires entre el 2009 y el 2012, documentos y una serie de estadísticas elaboradas por organizaciones sociales que luchan por los derechos de las personas trans y los resultados de la prueba piloto de la Primera Encuesta Nacional sobre la Población Trans: Travestis, Transexuales, Transgéneros y Hombres realizada por el INDEC - INADI durante 2012.

En su conjunto, este corpus presenta una serie de desafíos. El primero de ellos, en torno a la definición de los sujetos que son parte de este recorte, y la/as definición/es de la categoría "trans". Un segundo desafío se ubica sobre cómo transitan estos/as/xs estudiantes las instituciones educativas.

El artículo se organiza en tres apartados. En el primero de ellos avanzó sobre la categoría "trans" desde el discurso académico, normativo-legal, médico y del movimiento socio sexual con el objeto de problematizar en el contexto argentino su emergencia. En el segundo apartado me profundizó el análisis de la Viñeta 1 a partir de la pregunta: ¿Quiénes son estos/as/ $\mathrm{xs}^{3}$ estudiantes? Y por último, una de las primeras acciones

\footnotetext{
${ }^{3}$ La escritura de este artículo se encuentra interpelada por los usos apropiados del género gramatical ya que la regla estándar establece la generalización en masculino. Esta normativa, que ha sido fuertemente discutida desde los Feminismos y estudios académicos, ha sido modificada en la pragmática del lenguaje
} 
políticas educativas orientadas a la inclusión educativa: la Resolución 122 del gobierno de la Ciudad de Buenos Aires que permitía el cambio del nombre dentro de la escuela según la auto-percepción del/a estudiante. Esta resolución es anterior a la Ley de Identidad de Género y su implementación ofrece pistas para comprender mecánicas de exclusión/inclusión de los sujetos que permean actualmente la puesta en marcha de esta ley.

\section{Acerca de la categoría "trans"}

La obra de Michel Focault fue pionera al visibilizar los dispositivos sociales que configuran la sexualidad contemporánea. Esta lectura construccionista del poder en dialogo con los aportes de Judith Butler, Teresa de Lauretis entre otras/os ha discutido la naturalización del sexo/género. La categoría trans emerge en un escenario académico-político donde tres hipótesis respecto de la construcción identitaria sexo-genérica se ponen en debate.

Un primer momento donde la categoría género condensó los sentidos culturales, sociales, históricos que se construyen sobre una "base biológica" fija y determinada que nos divide en "hombres y mujeres". Un segundo momento, donde la misma categoría "sexo" es objeto de discusión y se visibiliza la misma capacidad reguladora de la categoría "género". Y por último un tercer momento, donde la revisión de categorías "estalla" y comienza una búsqueda de nuevas formas de nominar. Este hecho adquirió diversas tonalidades en distintos países, y se condensó con la gesta de leyes vinculadas a la identidad sexual/genérica (Belucci y Rapisardi, 2001).

La categoría "trans", que retoma este artículo, parte del desafío al sistema categorial sexogenero. Concretamente, esta publicación retoma la concepción de transgeneridad elaborada por Mauro Cabral como forma de vida que tiene en común el rechazo a la diferencia sexual como matriz natural y necesaria de subjetivación. El autor define con las siguientes palabras:

La transgeneridad constituye un espacio por definición heterogéneo, en el cual conviven -en términos no sólo dispares, sino también enfrentados- un conjunto de narrativas de la carne, el cuerpo y la prótesis, el deseo y las prácticas sexuales, el viaje y el estar en casa, la identidad y la expresión de sí, la autenticidad y lo ficticio, el reconocimiento y la subversión, la diferencia sexual y el sentido, la

mediante el uso del masculino y el femenino en cada oportunidad (por ejemplo, "los/las"). En el marco de este trabajo de investigación el uso de esa forma resulta estrecho dado que intención de es describir e interpretar las experiencias de jóvenes que cuestionan/transgreden las formas inteligibles de ser varón o mujer. Por esta razón, en el desarrollo del artículo la escritura oscilará entre el uso del "los/as" y la inclusión de la " $x$ ", que utilizaré para referirme a los/as/xs jóvenes trans. La intención final de esta decisión es que, aun entorpeciendo la lectura con sonidos poco reproducibles, podamos deshabitar las comodidades para dar lugar a una nueva producción de escucha, análisis e intervención. 
autonomía decisional y la biotecnología como instrumento que es, a la vez, cambio de batalla. (Cabral, 2007:1)

Esta nominación amplia, permite situar la experiencia de jóvenes que en su transitar discuten/cuestionan/transgreden las formas hegemónicas de "ser varón" o "ser mujer" en la actualidad. En este artículo, trabajo desde la propia percepción de los sujetos con quienes investigo; es decir, es a partir de su propia enunciación y no de una categorización externa de quienes son (o deberían ser). La categoría "trans" es también una forma "abreviada" de nominar al colectivo travesti, transgénero y transexual. Estas categorías identitarias son tributarias tanto de las luchas del movimiento sociosexual por políticas de reconocimiento (y redistribución) como también del sostenido trabajo académico. Vistas desde un nivel descriptivo de sus usos, estas categorías refieren a identidades que podrían reconocerse a partir de algunas características particulares. Aun cuando en su uso más corriente se usen de manera indistinta.

Desde el punto de vista político, cada una de estas categorías ha tematizado el espectro de cuerpos que en sus modos de vivir difícilmente puedan contar con un "closet" (Sedgwick, 1990). En este sentido, se trata de categorías que han irrumpido y han convocado a una renovada reflexión en torno a los "closet". A diferencia de otras maneras de vivir el cuerpo sexuado, quienes se reconocen a sí mismxs como travestis, transexuales o transgénero -o bien, como feminidades trans y masculinidades trans-, constituyen progresivamente en necesidad la posibilidad misma de tránsitos disímiles, de apariencias y estéticas que resultan difíciles de "ocultar". La existencia vital fuertemente se vincula a modos que traccionan enfáticamente el "ser mujer" y "ser varón", y que abre en el campo de la vida cotidiana la necesidad de reconocimiento. Para vivir dignamente resulta imposible construir un closet.

En su conjunto estas definiciones han discutido las concepciones brindadas por el campo de la medicina y psiquiatría para estas categorías. No reconstruiré aquí el campo de discusiones específicas, analizaré en cambio el caso de la nomenclatura utilizada en el Manual de Diagnóstico y Estadística de los Trastornos Mentales (en inglés: Diagnostic and Statistical Manual of Mental Disorders, -DSM-) de la Asociación Estadounidense de Psiquiatría (en inglés: American Psychiatric Association, -APA-)

El DSM contiene una clasificación de los trastornos mentales proporcionando categorías "claras" de diagnóstico para los profesionales de la salud. El manual tiene origen en 1952 y por medio de revisiones periódicas, quienes conformar la Asociación Estadounidense de Psiquiatría renuevan las nomenclaturas. El mayo del 2013 en su quinta edición, el manual descarta la transexualidad ${ }^{4}$ como enfermedad mental, conservando solamente la nominación "Disforia de género" ${ }^{5}$ como la angustia que sufren las personas que no se

4 Esta denominación incluye a diferentes formas de reconocimiento identitario: travesti, transexual, transgénero, feminidades trans, masculinidades trans.

5 Para una mayor precisión véase American Psychiatric Publishing (2013) 
identifican con el sexo asignado al nacer. Este cambio fue celebrado por organizaciones socio-sexuales, sin embargo, los reclamos de revisión persisten dado que tal clasificación continúa protocolizando la patologización de determinadas experiencias vitales.

Así por ejemplo, la Campaña "Stop Trans Patologización" ${ }^{6}$ (2013) señala cómo la categoría Disforia de Género continua sosteniendo la patologización que traía su forma de nominación anterior "Trastornos de Identidad de género" (DSM-IV) al vincular los procesos de tránsito en el género -necesariamente- a un sufrimiento o malestar. Por otra parte, los criterios de diagnóstico mencionan el término "incongruencia" lo cual es pasible de críticas pues se marca en el sujeto su supuesta incapacidad de mantener la congruencia.

Esta lucha por la despatologización de la vida de los sujetos se enmarca en una larga historia de reclamos que, en Argentina, contribuyó al cuestionamiento de prácticas represivas institucionalizadas. Los Códigos Contravencionales y de Faltas son un ejemplo de ello. Esta normativa es elaborada por cada legislatura distrital del país para regular las conductas que no se configuran desde la ley como delito pero si son pasibles de reproche legal. En la práctica cotidiana de autoridades policiales, no obstante, son utilizados como dispositivos de persecución y hostigamiento (Parchuc y Lumbertino, 2008).

El movimiento social trans ${ }^{7}$ ha denunciado de manera sistemática esta situación de continuo hostigamiento y violación institucionalizada. El Estado, de manera relativamente reciente, también ha comenzado a realizar acciones que acompañan estos pedidos ${ }^{8}$. La creación del Instituto Nacional Contra la Discriminación, la Xenofobia y el Racismo -INADI ${ }^{9}$ se orienta en tal sentido. En un informe ${ }^{10}$ realizado por este organismo analizando el uso de estos códigos advierte para el caso del Código Contravencional la Ciudad de Buenos Aires (Ley Nro. 1.477 -2004) que el Cap. II, Art. Nro. 81 es donde con mayor claridad se advierte la vulneración de los derechos de personas trans. El artículo regula la oferta y demanda de sexo en espacios públicos, aclarando también que "en ningún caso precede la contravención en base a la apariencia, vestimenta o modales" y que "la autoridad preventora sólo puede proceder al inicio de actuaciones por decisión de un representante

\footnotetext{
6 Se trata de un conjunto de organizaciones socio-sexuales que trabajan en pos de la igualdad y respeto del colectivo trans.

$7 \mathrm{Me}$ refiero a las distintas organizaciones socio-sexuales que luchan por los derechos, condiciones de vida e iguladad de personas que se reconocen como travestis, transexuales, transgénero o bien, en términos de feminidades trans o masculinidades trans.

8 Es indudable que el Estado es campo de lucha y disputa política, pudiendo albergar incluso acciones distintas para una misma población. Así por ejemplo, el Estado quién sostiene los Códigos Contravencionales y crea también, instituciones como el INADI

9 Este organismo estatal descentralizado es fundado, mediante la Ley Nro. 24.515 en el año 1995, iniciando su puesta en marcha dos años después. Entre sus objetivos principales se encuentra: elaborar y proponer políticas y medidas concretas para combatir la discriminación, diseñar e impulsar campañas educativas y comunicacionales, recibir y coordinar la recepción de denuncias, brindar un servicio de asesoramiento integral y articular acciones con otros organismos que trabajen contra la discriminación, la xenofobia y el racismo. 10 Informe del INADI (2008) sobre los Códigos Contravencionales y de Faltas.
} 
del Ministerio Público Fiscal". No obstante, de acuerdo al informe del INADI, en la práctica "real" este articulado ha sido utilizado para perseguir a personas trans (Parchuc y Lumbertino: 2008).

Otra vertiente por la que se deja entrever la trama de la patologización y criminalización ha quedado plasmada en la lucha jurídica que llevó adelante la Asociación de Lucha por la Identidad Travesti-Transexual -ALITT- para constituirse con persona jurídica frente a la Inspección General de Justicia ${ }^{11}$. Tras múltiples demandas con resultado negativo, finalmente la Corte Suprema en noviembre de 2006 resuelve otorgarle a ALITT la personería jurídica ${ }^{12}$. Este caso actualiza la disputa discursiva sexo-género en un plano concreto: el de las instituciones judiciales: ¿ALITT es una institución de "interés común"? (Ají de pollo: 2008:2009)

Por último, se puede observar el carácter patologizante-criminalizante y su lucha por el cambio en las marchas conocidas como "Marchas del Orgullo"13. Los lemas que embanderaron cada una de ellas en Buenos Aires son una puerta de entrada para dar cuenta de las demandas y sentidos que se construyeron desde los movimientos sociosexuales. La Marcha del Orgullo es organizada por una Comisión constituida por diversas agrupaciones socio-sexuales.

La Marcha del Orgullo combina "lucha y festejo" (Jones, Libson, Hiller, 2006) y reúne a gays, lesbianas, transgénero, travestis, transexuales, bisexuales, intersexuales y simpatizantes con la causa. Cada edición de la Marcha fue atrayendo un número mayor de concurrentes, así como también una expansión en actividades y consignas. En los últimos años, la Marcha comienza con una Feria del Orgullo desde el mediodía, donde distintas agrupaciones colocan stands en la Plaza de Mayo. A las 18hs. se inicia la caminata por Av. De Mayo hasta la Plaza de Congreso donde se culmina con un Festival Musical.

El lema central de cada una de las Marchas es fruto de negociaciones, a veces muy arduas, entre las distintas agrupaciones LGTBI. Este hecho, en ocasiones, se visibilizó en "Marchas" y "Contramarchas" simultánea, sobre las que no me detendré aquí. Los lemas consensuados hasta el 2014 recuperaron diversas consignas.

En la primera Marcha en 1992 se conjugan los reclamos a favor de la "igualdad, libertad y diversidad" en un remedo del lema de la revolución francesa pero con el tiempo las demandas adquirirán diferentes tamices. Mientras en algunos años, la lucha evidencia la

11 La posibilidad de contar con el estatus de persona jurídica habilita a las asociaciones -como ALITT- tener la capacidad para adquirir derechos y contraer obligaciones como institución.

12 Para una profundización sobre el caso la Agrupación Ají de Pollo ha publicado $(2008,2009)$ en dos tomos el conjunto de presentaciones, dictámenes y resoluciones.

13 Estas Marchas se realizan en distintas partes del mundo, y por lo general, el 28 de junio -fecha de los disturbios ocurridos en el Bar Stonewall Inn que ya he comentado en otros apartados-. En Buenos Aires la Marcha se realiza desde 1992; a partir de 1997 se traslada al mes de Noviembre en conmemoración al primer grupo homosexual y, en parte, también para evitar las bajas temperaturas. 
posibilidad de vida de los sujetos como los lemas de 1995, 1996, 1997 y 2009 donde la represión policial hacia personas transgénero, transexual y travesti se evidencia con expresiones como "La policía nos mata". Otros años, la lucha se ha organizado en torno a las demandas de ampliación de los derechos, ya sea por incorporación a la trama legal existente, cómo el caso de Matrimonio Igualitario o bien, creando legislación específica, como el caso de la Unión Civil o la Ley de Identidad de Género.

Un análisis de los lemas muestra que algunos tienen un carácter celebratorio en el reclamo, como por ejemplo en los lemas de 1997, 1999 y 2007. Los más problemáticos entre las agrupaciones han sido: "Toda la sociedad por el derecho a la diversidad" (2004) y "Somos todos maravillosamente diferentes" (2006). En ambas ocasiones, mientras que oficialmente se invitaba a la celebración de la diversidad y se propiciaba la ley de Unión Civil a nivel nacional (desde el 2002 vigente en la Ciudad de Buenos Aires), otras agrupaciones pedían la libertad gays, travestis y trabajadoras sexuales detenidas/os/xs en el contexto del Código Contravencional. El canto: "Se va a acabar / se va a acabar / la burocracia homosexual", sintetiza parte de los reclamos internos. (Jones, Libson, Hiller, 2006)

Estas distintas luchas se han plasmado en una serie de cambios específicos dentro del país. En esta dirección se destacan dos cuestiones que han innovado el campo legal (y con ello un nuevo estatus en la vida social): por un lado, la Ley Nacional de Salud Mental (Nro. 26.657) y por el otro, la Ley Nacional de Identidad de Género (Nro. 26.743).

La Ley de Salud Mental fue sancionada en 2010, y deroga una ley existente desde tal fecha (Ley Nro. 22.914). Este nuevo marco regulatorio incluye una mirada desde los Derechos Humanos, y concretamente, establece algunas definiciones importantes para el colectivo travesti, transexual y transgénero. Su Art. 3ro. Define el marco de la ley, reconociendo a la salud mental como un proceso de distintos componentes: históricos, socio-económicos, culturales, biológicos y psicológicos, cuya "cuya preservación y mejoramiento implica una dinámica de construcción social vinculada a la concreción de los derechos humanos y sociales de toda persona". Explícitamente agrega que en ningún caso el diagnóstico en salud mental puede efectuarse sobre la base exclusiva de: "d. elección o identidad sexual", entre otros. Esta distinción discute con la posibilidad de uso de los manuales de diagnóstico que he descripto en párrafos anteriores e implica un avance a nivel internacional en términos de la lucha del movimiento socio-sexual en la medida que retira el estatus de enfermedad.

La Ley de Identidad de Género votada en mayo del 2012 dispone que las personas que así lo elijan puedan ser inscriptas con el nombre y sexo según su propia auto-percepción. Es decir, la ley permite el cambio de los datos registrales a partir de la demanda de las personas, sin necesidad de diagnósticos o cualquier otro tipo de certificación. La ley define la Identidad de Género en los siguientes términos: 
Art.2do - Definición. Se entiende por identidad de género a la vivencia interna e individual del género tal como cada persona la siente, la cual puede corresponder o no con el sexo asignado al momento del nacimiento, incluyendo la vivencia personal del cuerpo. Esto puede involucrar la modificación de la apariencia o la función corporal a través de medios farmacológicos, quirúrgicos o de otra índole, siempre que ello sea libremente escogido. También incluye otras expresiones de género, como la vestimenta, el modo de hablar y los modales.

La enunciación es amplia y toma como fundamento principal "lo sentido por la persona". En función de ello en el Art. 4to. establece una serie de requisitos para la rectificación registral del sexo, el cambio del nombre e imagen. Se distingue en primer lugar la acreditación de una edad mínima de 18 años (otros casos están regulados por el artículo 5 , del cual haré alusión más adelante), en segundo lugar, la presentación de esta solicitud en el Registro Nacional de Personas, y por último, la expresión de un nombre de pila.

La normativa señala que en ningún caso será requisito "acreditar intervención quirúrgica por reasignación genital total o parcial, ni acreditar terapias hormonales $\mathrm{u}$ otro tratamiento psicológico o médico". Esta definición resulta novedosa ${ }^{14}$ en la legislación específica sobre el tema. La ley argentina inaugura un estatus legal para los sujetos solicitantes amparado su derecho de identidad sobre el propio reconocimiento, sin solicitarse diagnósticos, tratamientos, testimonios o informes de terceros como lo plantean otras leyes vigentes en el mundo. En su Art. 5to., la ley incluye los modos de aplicación para menores de 18 años, lo que marca nuevamente una diferencia con las otras normas al considerarse tal posibilidad. Para los/as/xs jóvenes y niños/as/xs se prevé que el cambio puede realizarse contando con la compañía de sus tutores legales y el expreso consentimiento del interesado/a/x. En caso de encontrarse en desacuerdo sus representantes legales, puede asumir ese rol un juez. Esta definición que a nivel internacional también resulta innovadora generó una serie de debates locales.

En primer lugar lo estipulado por la ley en su Art. 5to., desde el punto de vista legal dialoga con el concepto de la autonomía progresiva de niños, niñas y adolescentes regulado por la Convención de Derechos del Niño. Viola señala que

Esto implica reconocer que todos los niños, tanto los pequeños como los adolescentes, son los protagonistas de sus propias vidas, sin desconocer que esa autonomía irá variando de acuerdo a la etapa evolutiva en la que el niño se

14 Por ejemplo, antecedentes legales en otros países recuperan otros parámetros, la Ley 3/2007 "Reguladora de la rectificación registral de la mención relativa al sexo de las personas" (España) y la Ley Nro 18.620/2009 "Derecho a la identidad de género y al cambio de nombre y sexo en documentos identificatorios" (Uruguay) son ejemplo de ello. En estas regulaciones se exige el diagnóstico médico-psiquiátrico y la adecuación entre nombre y sexo. 
encuentre. De lo contrario, otorgarles autonomía a los niños sin considerar sus posibilidades evolutivas implicaría dejarlos en un estado de desprotección. (Viola, 2012: 87)

La Convención, que se encuentra incorporada a la Constitución Nacional y, posteriormente, se materializa en la Ley Nro. 26.061 de Protección Integral de los Derechos de Niñas, Niños y Adolescentes, explícita el derecho de los niños y niñas a ser oídos en todos los ámbitos, y particularmente, en "los asuntos que les conciernen y en aquellos que tengan interés", progresivamente en función de su madurez. Esto implicaría la posibilidad de matizar lo estipulado en el Art. 5to. de la Ley de Identidad de Género.

Esta nueva mirada legal sobre los niños, niñas y adolescentes los ubica como sujeto de derecho. Sin embargo, en la trama legal actual, también persisten normativas atinentes a concepciones anteriores. El punto más controversial reside en la vigencia del Código Civil que establece una capacidad jurídica nula en el caso de menores de 14 años y una capacidad relativa en menores de 14 a 18 años. Desde este ángulo, el artículo 5 brinda la posibilidad a niños/as y adolescentes, no obstante, la figura del tutor/juez la emplaza en un marco más "tradicional" del derecho.

En segundo lugar, la estipulación del Art. 5to. parece ir a "contra mano" de la realidad fáctica. La expresión de la identidad no se encuentra vinculada a una edad específica. Un caso que tomó estado público durante el 2013, invita a una nueva lectura respecto de la mecánica final que instaura el artículo. El caso de "Lulu", una niña de cinco años para la cual se pidió el cambio de DNI. Siguiendo lo establecido en el Art. 5to, esta niña en compañía de sus tutores -en este caso padre y madre- solicitó tal trámite en el Registro de las Personas de la provincia de Buenos Aires, siendo denegado en 3 ocasiones.

Acompañada la familia desde la organización Comunidad Homosexual Argentina -CHA-, se iniciaron pedidos públicos en diarios y por medio de cartas a distintos representes políticos (presidenta, gobernador). La resolución no resultó inmediata, en parte por lo novedoso del caso, que hizo que la falta de experiencias previas generara incertidumbres administrativas, por ejemplo. Salvados los imprevistos, no obstante, se presentaron una serie de cuestionamientos respecto de cómo proceder, aun cuando el artículo ya lo fijaba.

Finalmente, mediante una presentación de la Secretaría Nacional de la Niñez al Registro de las Personas bonaerense, y con la participación del Poder Ejecutivo Provincial, se accedió al cambio registral de los datos de Lulú. Sin necesidad de judicialización, la niña obtuvo su nuevo DNI y partida de nacimiento, dejando un antecedente para futuros pedidos. Este proceso, si bien de resolución positiva, permite dar cuenta de la vaguedad del artículo y sus resistencias institucionales. (Mansilla, 2015)

Otro articulado de la ley que reviste de especial atención para el ámbito educativo, es aquel que establece el "trato digno" (Art. 12). Se establece, que "se deberá respetar la 
identidad de género adoptada por las personas, en especial por niñas, niños y adolescentes, que utilicen un nombre de pila distinto al consignado en su documento nacional de identidad". Al solo requerimiento del interesado/a, este cambio debe darse tanto en los modos de citación, registro, legajo, llamado o cualquier otra gestión tanto en ámbitos públicos como privados. Se prevé asimismo que cuando sea necesario registrar los datos del DNI, se utilizarán las iniciales del nombre y apellido completo, día y año de nacimiento, junto el nombre elegido por el/la solicitante.

Este acápite permitió vislumbrar el paso de un período del reclamo por la fuerte persecución policial, patologización y criminalización donde los códigos contravencionales o manuales de diagnóstico signaban la experiencia de las personas trans, a una nueva etapa. En ella, el reconocimiento legal como sujetos con derechos se abre, organiza una inteligibilidad novedosa pero también heredera de una historia donde la vida siempre estuvo en peligro. Estas notas permiten inscribir, y avanzar en la compresión de un estudiantado frágil, donde la inclusión escolar es puesta en jaque.

\section{¿Estudiantes?}

La viñeta 1 con que inicié este artículo presenta desde la voz de una asesora pedagógica, la descripción de una escena áulica que la deja perpleja: "se confundió" un estudiante "varón" con una "mujer" al tener sus "ojos delineados". Durante mi trabajo de campo resultó imposible conocer la voz del/a estudiante dado que se "pierden los rastros de él/ella" en la escuela. Este acontecimiento se constituyó en un indicio.

En la escena descripta se despliega en simultáneo la vida en singular de un estudiantes leída por una adulta y a su vez, se evidencia cierto correlato con otros relatos. Esta escena se inscribe en un cuerpo de relatos breves y fragmentarios, junto con otras historias de chicos que dejan la escuela. A lo largo del trabajo de campo fui recogiendo distintas referencias sobre relatos similares: "había un chico, pero era difícil con el tema del baño. Fue difícil, queríamos que se quedará pero también es difícil por el tema que esta escuela es de noche y ella lo necesita para trabajar" (director de una escuela pública secundaria de la Ciudad de Buenos Aires).

Un profesor de otra escuela comenta: "él chico se pasó a la noche, a la de adultos, con gente grande que entiende más" o en palabras de una estudiante de otra institución: "yo tengo un amigo, a la escuela nada, va de chico pero cuando salimos es una reina" o bien, desde una colega que trabaja dando cursos de ascenso y una tarde me consulta: "tengo una vice en el curso que está preocupada porque tiene una chica travesti y no sabe cómo hacer, ¿podrás entrevistarte con ella?". Al comunicarme con ella me comenta: "ya no viene más ese chico".

Esta colección de relatos breves adquiere otra densidad al leerse bajo la grilla de otros trabajos que han visibilizado la experiencia de estos/as/xs jóvenes. Los aportes realizados por Josefina Fernández en las últimas décadas en Argentina, arrojan aristas desde donde 
situar esta sucesión de relatos. Esta autora, indaga los procesos de construcción de la identidad travesti en el ámbito de la Ciudad de Buenos Aires partiendo de la hipótesis que "el travestismo cuestiona los principios de clasificación y reconocimiento de identidades de género legitimadas socialmente" (cursiva de la autora) (2004:16).

A partir del testimonio de un conjunto de travestis jóvenes y adultas, la autora analiza diversos ámbitos donde la construcción de la identidad de sus entrevistadas se pone en juego: la familia, el trabajo (y con ello la prostitución), el espacio público y el propio cuerpo. Fernández (2004) señala cómo aparece mencionada la escuela en estos relatos. Una primera mención de la institución escolar emerge en el relato de las travestis entrevistadas cómo una de las primeras fuentes de reconocimiento de la existencia de "dos sexos" -y de dos géneros-. Fernández (2004) trae el recuerdo de una de ellas: el comienzo del jardín infantes y el momento en que la preferencia por un color impropio para un niño el rosa marcaron su condición de diferencia. Junto con la familia, la escuela resulta escenario privilegiado para las entrevistadas, lugar donde los primeros conflictos se tornaron visibles. La discriminación y el pesar generados por los propios/as compañeros/as, la violencia de docentes (en particular en torno a las prácticas deportivas) persisten en el testimonio de estas travestis adultas al rememorar la cotidianidad escolar. Una segunda mención de la escuela acontece en torno a la expulsión y/o auto expulsión escolar, y en este sentido las travestis entrevistadas por Fernández "atribuyen a la escuela la responsabilidad de un presente marcado por la pobreza y la marginación" (82:2004). En esta dirección, la escuela no solo resultó un ámbito desde donde tramitar penosamente la propia identidad sino también es vivida como factor que ha condicionado la situación actual de vida. Una tercera mención aparece en una nota a pie de página -en lo que tal vez resulte una de las primeras aproximaciones numéricas al tema- donde se considera la situación de las travestis en la Ciudad de Buenos Aires, señalándose:

El nivel educativo de las travestis fue explorado por la Defensoría del Pueblo en su Informe preliminar sobre la situación de las travestis en la Ciudad de Buenos Aires. Según este informe, sobre una muestra de 147 travestis, la secundaria incompleta es el máximo nivel de estudios que posee el $50 \%$ de las entrevistadas. El 19\% sostuvo haber finalizado sus estudios primarios, mientras que sólo el $13 \%$ de la muestra completaron el nivel secundario. (Fernández, 2004:83)

El estudio citado fue realizado en el 1999 desde la Defensoría del Pueblo, titulándose: "Informe preliminar del estado de situación de las travestis en la Ciudad de Buenos Aires". Y se constituyó en referencia para otras demandas, entre ellas una serie de medidas a favor del respeto de nombre de pila, que finalmente se logró bajo Resolución Nro. 122 en 2003 (sobre ella se avanzará en el próximo apartado).

El conjunto de "relatos breves" de estudiantes que ya no están en la escuela, tienen un correlato estadístico. Desde distintos actores sociales se construyeron una serie de datos estadísticos que permiten contextualizar, o más bien, interpretar las ausencias de 
estxs/as/os jóvenes; o bien, sus dificultades para transitar la escuela común. La más recientes de las propuestas fue la realizada por el INDEC/INADI: Encuesta Nacional sobre la Población Trans: Travestis, Transexuales, Transgéneros y Hombres Trans. (2012) (Prueba piloto). ${ }^{15}$

Tras la sanción de la Ley de Identidad de Género, durante 2012, el Instituto Nacional de Estadística y Censos (INDEC) en convenio con el Instituto Nacional contra la Discriminación, la Xenofobia y el Racismo (INADI) realizaron de forma conjunta la Primera Encuesta sobre Población Trans $^{16}$ con el objeto de contar con información sociodemográfica de una población "notablemente vulnerada, tanto desde el punto de vista social como del ejercicio de los derechos ciudadanos" (2012: 2), generando datos a nivel nacional y desde un organismo gubernamental.

Identificando como "población trans" a aquellas personas que se auto-perciban ${ }^{17}$ como "travestis, transgénero, transexuales, feminidades trans, masculinidades trans", la encuesta explora las condiciones de la vida cotidiana así como también indaga acerca de las experiencias de discriminación, alfabetización jurídica y acceso a la justicia. Para ello, el estudio propuesto se organizó en tres etapas: 1 . Prueba de Cuestionario, 2. Prueba Piloto en La Matanza y 3. Realización de la encuesta a nivel nacional. A continuación presentaré algunos de los hallazgos encontrados durante la segunda etapa ${ }^{18}$.

La prueba piloto fue realizada en el partido de La Matanza (provincia de Buenos Aires), y por medio del contacto con organizaciones sociales vinculadas a la población travesti, transexual y transgénero se llegó a los/as primeros/as entrevistados/as. Luego estos brindaron datos de otros contactos posibles, llegando constituirse una muestra con 209 participantes.

Estos datos permiten construir un mapa educativo más amplio, en la medida que visibiliza a determinados sujetos que habitualmente han sido cuantificados bajo la clasificación sexo tradicional: "varón" o "mujer" en los registros oficiales. Uno de los primeros datos que aporta la Encuesta a Población Trans en su etapa de piloto, y que sigue la línea de lo ya presentado, es la indagación por el máximo nivel escolar alcanzado. Un tercio de los/as encuestados/as ha transitado el nivel secundario (o polimodal) de manera inconclusa

15 Otras encuestas que marcaron un hito fueron: Encuesta a Población Travesti en Ciudad de Buenos Aires/Buenos Aires (2005) y la Encuesta Nacional a Población Travesti (2007) ambas impulsadas por organizaciones trans.

16 Informe Prueba Piloto, Primer Encuesta a la Población Trans (INDEC, 2012)

17 Cabe aclarar que este artículo boga a favor de la auto-percepción/reconocimiento del sujeto como criterio de designación de la identidad. Es por ello que no se definen las categorías "travesti", "transexual" o "transgénero" dado que ello recaería en el establecimiento de criterios externos a la persona. El punto en común de estas experiencias reside en el hecho de la asignación de un sexo/género al nacer que posteriormente es vivido de manera diferente a la socialmente establecida.

18 A la fecha de la escritura de este artículo, solo se encontraban disponibles los resultados de la Prueba Piloto. 
mientras que menos de la mitad $(45,4 \%)$ solo recorrió el nivel primario. Solo el $14,8 \%$ tiene la secundaria o polimodal completos.

Además de indagar variables socio-demográficas, la encuesta, explora percepciones en torno a la discriminación. Al preguntar por las vivencias de discriminación por parte de compañeros/as en la escuela, la respuesta resulta dividida: mientras 4 de cada 10 trans masculinos han vivido tales experiencias, en el caso de las trans femeninas asciende a 6 de cada 10. Por último, es sugerente la edad en qué los/as encuestados/as señalan como inicio de la expresión de su identidad de género. La categoría que recoge mayor frecuencias es "entre los 13 años y 17 años", hecho que coincide con la escolarización secundaria (47,5\%). Hasta los 12 años, un 32,5\% de los/as encuestados/as, un 15,8\% entre los 18 y los 25 años y un 3,8\% después de los 26 . Estos datos refuerzan la necesidad de revisión de los mecanismos de implementación de la Ley de Identidad de Género, pues el $80 \%$ de los/as encuestados/as expresa su identidad en una edad inferior a la establecida -18 años-. Y si bien, se establecen mecanismos a los que pueden apelar niñas, niños y jóvenes no podemos olvidar que son obligados a la buena voluntad de su tutor/a, en gran medida.

Ahora bien, los/as estudiantes que son parte de este artículo comparten entre sí un vínculo distinto con los closet: para ellos/as/xs no es una posibilidad. La expresión de su propia voz los/as vincula con estas disputas de manera más expuesta. Esta hipótesis nos brindaría una explicación a los procesos de abandono de la escuela secundaria desde la perspectiva de los sujetos, poniendo sobre el tapete las formas que adquiere la inclusión en este nivel.

A su vez, esta interpretación logra mayor sustentabilidad cuando es conjugada con una serie de medidas provenientes de la política educativa, las que han intentado avanzar en modificaciones o abriendo nuevas propuestas para la inclusión de estos/as/xs estudiantes. Ya no se trata, entonces, de las posibilidades de los sujetos para negociar, resistir, apropiarse de un formato escolar que reitera la clasificación de los cuerpos sexuados, sino también, de otras formas de situar ese formato para darle lugar a tales experiencias. En el próximo apartado se avanzará sobre ello.

\section{Formas de incluir. El nombre propio, una forma de interpelar el formato común.}

El armado de nuevas propuestas para la inclusión de estos/as/xs estudiantes posibles deja al descubierto los límites del propio sistema en general y del formato escolar secundario común en particular. Una de las primeras acciones político-pedagógicas que instalaron en la escena social-educativa la necesidad de repensar a estos/as/xs jóvenes en la escuela y cómo comenzar a dar respuesta su exclusión sistematizada fue la Resolución Nro. 122 del Gobierno de la Ciudad de Buenos Aires que permite el cambio de nombre de pila. 
Parte de los informes estadísticos que presentaba en el apartado anterior junto con la interpelación de Lohana Berkins en ocasión del pedido a la institución donde estudiaba para docente del nivel primario el cambio del nombre de acuerdo a su auto-designación, Daniel Filmus - Secretario de Educación del Gobierno de la Ciudad- firmó la resolución Nro. 122 que recomienda "a los establecimientos educativos de la CABA y a las dependencias de la Secretaría de Educación, que se garantice el respeto por la identidad de género, dignidad e integración de las personas pertenecientes a minorías sexuales".

Sin otra especificación a la ya expresada, la resolución presenta una innovación legal sin precedentes en el país. Sin embargo, tal formulación encerró una serie de dificultades para su concreción. Con el tono de recomendación de "garantía de respeto por la identidad de género", se irrumpe en la escena social visibilizando el derecho al nombre propio, aunque no se explicitan los mecanismos por los cuales debería hacerse efectivo en lo cotidiano. En este sentido, la resolución puede interpretarse como un deseo declarativo más que una nueva forma de regulación social. La novedad en su contenido, difícilmente encuentre en el campo social mecanismos desde donde instalarse, pudiendo avizorarse lugares de resistencia. El tiempo, sin embargo, marcó cómo esta resolución firmada en el 2003 fue parte de la sanción de la Ley de Identidad de Género en el 2012.

Durante el 2009, seis años antes de la sanción de la ley y en plena vigencia de la resolución, Ale cursaba el 6to. año de una escuela pública prestigiosa e intentaba hacer uso de la misma. Su recorrido permite problematizar desde otro ángulo, la cuestión de la presencia de estudiantes trans en la escuela común. Ale vivía con sus padres en Vicente López. Su padre y madre profesionales, universitarios, de "clase media". En una entrevista, relata:

Ale: Yo llegue a ver la resolución de Lohana, y la traje a la escuela para que vean el tema del nombre. Sería lo correcto en la escuela al menos mostrar tolerancia. ¿No? Por eso yo la traje, para que al menos eso.

Entrevistadora: ¿Y qué paso?

Ale: Me dijeron que no la podían aplicar.

Entrevistadora: ¿Y sabes por qué?

Ale: Decían, que esa es una resolución de la Ciudad de Buenos Aires, y este no es un colegio de la capital, sino de la Universidad. Y la Universidad, la universidad.

Entrevistadora: Ajá.

Ale: Y la rectora, me dijo que estaba bien, que le parecía bien, pero que no podía aplicarla por este tema. Ella apoyaba el cambio, pero no lo podía aplicar.

(Entrevista, Abril de 2010)

La resolución invita pero no obliga, dejando un intersticio a la buena voluntad de las instituciones -establecimientos educativos tanto de gestión privada como estatal, así como también, instancias administrativas de la Secretaría de Educación- garantizar el respeto a la identidad de género. En este caso, la jurisdicción en la que se domicilia la 
escuela resultó un ámbito de "no aplicación" desde lo narrado por Ale. Esta razón se superpone con la opinión contraria de la rectora que sí apoyó el pedido de Ale. La justificación se asienta en el emplazamiento de la escuela que aun estando ubicada en la Ciudad de Buenos Aires al depender de una universidad arroga para sí autonomía en la aplicación legal.

El relato sorprende. Y pronto se sospecha en tal justificación otras imposibilidades normativas ya no asentadas en el juego legal jurisdiccional, sino en los vericuetos espaciales de la norma de lo inapropiado. Es decir, se vislumbra un artilugio para no dar lugar a algo que es percibido desde el mundo adulto como inapropiado: "nacer mujer y querer tener un nombre de varón". Producto social, que supera las voluntades individuales, este mecanismo artimañoso, evidencia lo inapropiado de lo demandado.

Siguiendo las palabras de Donna Haraway, "Ser inapropiado/ble es no encajar en la taxón, estar desubicado en los mapas disponibles que especifican tipos de actores y tipos de narrativas, pero tampoco es quedar originalmente atrapado por la diferencia." (Haraway, 1999:129). En este juego entre inapropiado y diferencia que la autora explora, lo inapropiado porta de potencial político (y poético) para la configuración de identidades que escapen a los binarismos modernos. No encajar es desubicarse incluso respecto de la diferencia. Lo inapropiado guarda para sí, la posibilidad de la licencia poética y creatividad. Ale describe su tránsito por la escuela:

Entrevistadora: Y hasta ahora, en la escuela, ¿cómo te llamaban?

Ale: Acá en la escuela tenía un apodo híper neutro y con eso, estaba bien. Y un día le dije a mis amigos que me llamen por mi nombre nuevo. Pero con el apodo estaba bien. No era ni una cosa, ni otra.

Entrevistadora: ¿Qué cosa y qué otra?

Ale: (ríe) ni de varón, ni de mujer.

Entrevistadora: Claro.

Ale: Después les dije a mis amigos lo de "Ale", y empezaron ellos. A veces me da miedo, por el trabajo, con la familia. Pero me da miedo cambiar el nombre, el nombre ¿no? Da miedo, sería más fácil si fuera mujer como dice el documento. A veces pienso, si alguien me viene a robar y se da cuenta que soy... tal vez sería peor, ¿no? (Entrevista, Abril 2010)

Un apodo "híper neutro", un diminutivo neutro también, son las formas habituales con que llaman a Ale. Creaciones diminutas que irrumpen la cotidianidad y al mismo tiempo, se mimetizan en el paisaje. ¿Quién desconfiaría de un/una "Ale"? Su deseo era "ni una cosa, ni la otra", ni varón, ni mujer aclara luego. Sin embargo, pronto aparecen en relación a este deseo, temores. ¿Qué pasará en el trabajo? ¿Qué pasará con la familia? ¿Qué pasará en un posible robo? Sin reducir cada uno de estos ámbitos, pero si visibilizando un punto de encuentro se podría preguntar: ¿qué pasará frente a un "Otro"?. La escuela es nuevamente, un ámbito donde plantearlo: 
Ale: Primero lo planteé acá en la escuela esto, y después en mi familia. Primero traje la resolución de Lohana (Resolución 122) acá, hable con mis amigos de la escuela, con ellos comencé con el nombre: Ale. Después hable con mis papás, les escribí una carta. Les puse mucho que los quería, todo el tiempo les escribí que los quería y que me estaba pasando esto.

Entrevisadora: ¿y cómo te fue?

Ale: Bien. Al principio se quedaron helados, igual se lo imaginaban, y después me apoyaron.

Entrevistadora: ¿Cómo?

Ale: Mi papá vino conmigo a la escuela para charlar lo que yo necesitaba. Y la rectora organizó una reunión con los profesores. Ahí acordamos oralmente que me llamaran con este nombre. Pero no podía estar en los papeles. Yo supongo porque le tenían miedo al rector de la UBA. Igual era raro, le cambiaron el nombre a una chica con apellido chino porque no se podía pronunciar y ahí lo cambiaron en los papeles y todo. Y a mí no. Ella me contó, todo.

Entrevistadora: Ajá.

Ale: Sí, viste, ¿̇no? Los profesores estaban muy preocupados por si era varón o mujer y le decían a mi papá: "Pero ¿qué es varón o mujer?" Los profes querían saber eso. "iCual era la verdad!" Y yo no quería que se dijera así, no es tan así. Y querían que mi papa dijera que era.

La escuela resulta para Ale un primer espacio donde nombrarse. La resolución en esta secuencia resulta ser una excusa eficiente para poder plantear lo inapropiado del pedido de Ale. Paradójicamente la escuela en este relato adquiere una doble tonalidad. Por un lado, como espacio propicio donde apelar, pedir y hablar de esto y en simultáneo, como ámbito donde el imperativo de certeza se pone en marcha. Descartadas las posibilidades de aplicación, la alianza entre rectora, padre, profesores y estudiante habilita la posibilidad de una reunión para hablar sobre el pedido de Ale. En esa misma conversación, el imperativo con tamiz amigable de "saber con exactitud quién es Ale", saber específicamente si es "Mujer" o si es "Varón" se despliega en la escena. La nueva escena se monta entonces: la escuela incorpora la demanda del estudiante. Por medio de un acuerdo verbal, se establece que su nombre de pila será Alejandro o bien su apellido. No obstante, la incorporación queda encorsetada en la necesidad, desde los/as docentes de definir parámetros concretos respecto de la identidad sexo-genérica.

Por otra parte, en el relato del estudiante aparece un nuevo contraste: a diferencia de su caso, se logró cambiar el apellido y nombre de una estudiante por ser los mismos "impronunciables". Un apellido/nombre que no se puede pronunciar en el español a causa de su origen chino aparece en las listas de estudiantes de la escuela, sin embargo, su pedido de cambio de un nombre solo pudo canalizarse por medio de una reunión con resultado de "acuerdo oral". En el cotidiano, este acuerdo diferenciará posturas entre el cuerpo docente: 
Entrevistadora: ¿Y qué paso en la escuela después?

Ale: Variado, algunos me decían "Ale" o "Alejandro" que era el nombre que había elegido. Por ejemplo, en un trabajo práctico para psicología en grupo, todos pusieron nombre y apellido y yo solo el apellido. La profesora se dio cuenta, entrego el trabajo y me miró, yo fui después le pedí disculpas, y hablamos. Ella me pregunto, bien. No sabía, lo de mi nombre, y me preguntó como quería hacer. Y, entonces quedamos con el nombre Alejandro. Ella se dio cuenta del detalle: de que yo no puse el nombre. Para mí fue importante. El acuerdo fue oral, así que todos los documentos como la lista quedó mi nombre legal. Otros profes, en cambio, cuando pasaban lista y decían cada nombre y cuando llegaba el mío, lo leían y me miraban, y me volvían a mirar. Algunos parecía que lo hacían a propósito. Decían el nombre legal así bien pronunciado, usaban a mi nombre legal de una manera, así así. Justo a mí se les ocurría decir por el nombre legal. A todos los demás apellido y a mí se les ocurría llamarme por mi nombre legal. Justo a mí.

El abanico queda dibujado entre aquellos/as docentes capaces de detenerse en el "detalle" y otros/as, donde la pronunciación del nombre legal adquirirá para Ale cierta sospecha sobre la emergencia de formas de discriminación. Incluso Alejandro así lo afirmaba:

Ale: Cada tanto, yo escuchaba pero no escuchaba. No es tan abierta la discriminación. Hablan atrás tuyo, vos escuchas pero no escuchas. Raro, ¿̇no?

Entrevistadora: Ajá.

Ale: cada tanto un "puto", pero te reís de eso. Yo ahora me identificó como un nombre trans, no me identificaba como mujer pero tampoco como varón. Yo me quería cambiar el nombre.

No sé si soy un varón...o soy mujer...se puede tener nombre de varón y no ser varón. $Y$ ahora me identifico como varón trans. Pero en realidad ya ahora no me importa tanto que soy. Soy yo, y listo.

Ese hablar "por detrás", esas categorías de las cuales finalmente Ale aprendió a reírse, un deseo de no clasificación acontecen entre sus palabras, una posibilidad de ser quien se es "y listo", en una trama incierta de taxonomías. Tal como afirmará Haraway (1999) los cuerpos biológicos diversos emergen. La emergencia, sin embargo no resulta ingenua. La posibilidad creativa de estos cuerpos biológicos diversos acontece en una grilla socialmente organizada. La escuela, un dispositivo de ello (Lopez Louro, 2010).

El pedido de Ale pone en tensión el formato de la escuela común para abordar lo inapropiado. La respuesta resulta paradojal, habilita pero encorseta. Ale va a una escuela en la que asisten sectores de recursos económicos medios y altos-medios, sus padres pertenecen a la generación "progre" respecto a algunas temáticas -que acompañan al estudiante en sus reclamos- y la propia escuela es reconocida como institución formadora 
y eventualmente "innovadora". Una de las primeras instituciones en plantear la necesidad de educación secundaria mixta, por ejemplo.

El caso permite vislumbrar cómo una multiplicidad de factores hacen posible o no, o más bien, imprimen características particulares a la experiencia cotidiana de los/as estudiantes. La posibilidad de contar con recursos materiales y simbólicos diferentes habilita también, interpelaciones distintas a un formato escolar que tampoco es inamovible. La posibilidad exitosa de este estudiante (si éxito se mide en la posibilidad de permanecer y egresar de la institución) no invisibiliza las dificultades de otros/as. Más bien, amplifica en el espejo las condiciones de esos otros/as.

\section{Desigualdades sexuadas: la escuela secundaria y los desafíos de la Ley de Identidad de Género}

Analizar las formas de transitar la escuela secundaria de jóvenes trans que se reconocen como travestis, transexuales, transgéneros, feminidades o masculinidades trans, tal como se anticipó, reviste un conjunto de reparos, tensiones y paradojas en torno a los obstáculos que implica para estos/as jóvenes los trayectos escolares.

Un primer reparo, es el encuadre desde cual se sitúan el análisis de los recorridos, sentires y viviencias de estos/as/xs jóvenes. Su singularidad no puede ser desgajada de la inscripción en una trama de las relaciones sociales, histórica y culturalmente situadas. La experiencia no es una evidencia transparente, por el contrario, se construye desde un posicionamiento social del sujeto y siempre es una interpretación que requiere de otra lectura (Scott, 2001). En este sentido, las experiencias son del sujeto $y$, al mismo tiempo, son del mundo social, donde el conflicto y la frontera se hacen presentes (Anzaldúa, 1987). La experiencia no es producto unívoco (y lineal) del orden social imperante y, a su vez, ese orden social no es totalmente uniforme. Es por ello que la experiencia se origina entre elementos culturales flojamente acoplados y la negociación es parte de ella (Rockwell, 1996).

Un segundo reparo reside en quiénes se atribuyen la posibilidad de interpretación de estas vidas. Históricamente la psiquiatría y la medicina se han constituido en la voz autorizada para enunciar estas identidades y desautorizar la propia voz de los sujetos sobre sí mismos. La academia y sus estudios también se han arrogado para sí, la "interpretación crítica" de unos y otros. En este marco, resulta urgente construir soportes que permitan fortalecer y potenciar las propias interpretaciones de los sujetos. 
Teniendo presente estas cuestiones, este trabajo exploró desde una serie de entrevistas, recortes periodísticos y documentos estadísticos el paso de los/as/xs jóvenes trans por la escuela secundaria. Para ello resultó imprescindible recuperar algunos hitos que permiten situar la categoría trans en el debate social y local. En esta dirección, la Ley de Identidad de Género reposiciona sentidos e interpela a la escuela hoy. Conocer estas experiencias juveniles recobra nuevos sentidos: esta ley se implementa en terreno social fuertemente marcado por estereotipos, y la escuela no escapa a ello.

Tal como pudimos analizar, los procesos de enunciación -con mayores niveles de visibilización- de un cuerpo sexuado que no se acomoda a lo ya establecido en la grilla de requisitos de "ser varón" y "ser mujer", aparecen mayores obstáculos para permanecer e incluso se llega a la exclusión; en términos estadísticos podría describirse como exclusión sistematizada: no se trata de un/a estudiante sino de un colectivo que no accede a un trayecto escolar que actualmente es obligatorio en el país.

La exclusión del sistema educativo o, desde una lectura individualista, el abandono del estudiante es moneda corriente. La configuración de proyectos como la Resolución Nro. 122 es una respuesta que nos obliga a la reflexión en torno a los parámetros de la inclusión en la escuela secundaria común.

La desigualdad por la que transitan estos/as jóvenes no solo se traduce en esta exclusión, sino también en lo que significa esta exclusión para el conjunto del estudiantado: la enseñanza de una jerarquía de posiciones ciudadanas. En esta dirección, la experiencia educativa de estos/as/xs jóvenes se encuentra regulada por las formas de hacer escuela y, al mismo tiempo, estas formas de hacer se encuentran interpeladas a partir de la presencia de estos/as/xs estudiantes. En este juego no solo participan los/as/xs protagonistas, sino la comunidad educativa completa.

En esta escena escolar, la Ley de Identidad de Género es una herramienta que apuesta al reconocimiento desde la propia percepción y la constitución de relaciones marcadas por el trato digno. Esta apuesta implica desafíos profundos que no solo se dirigen a imaginar una escuela donde la capacidad de recepción y sus mecanismos de expulsión sean interrogados sino, donde se revisen las tramas de poder que instituyen jerarquías ciudadanas en las aulas. Es allí donde una real aplicación de la Ley de Educación Sexual Integral parece tornarse urgente: reconociendo que si toda educación es sexuada (Morgade, 2011) es necesario recomponer políticas públicas que organicen y produzcan una escena escolar más justa y democrática para quienes la recorren. 


\section{BIBLIOGRAFÍA}

AAVV (2013). Reflexiones de STP sobre el proceso de revisión de la CIE y la publicación del DSM-5 STP, Campaña Internacional Stop Trans Pathologization Disponible en: http://www.stp2012.info/Comunicado_STP_agosto2013.pdf

Agrupación Ají de Pollo (2008). Políticas de Reconocimiento I.

Agrupación Ají de Pollo (2009). Políticas de Reconocimiento II.

American Psychiatric Publishing (2013). Gender Dysphoria En: http://www.dsm5.org/Documents/Gender\%20Dysphoria\%20Fact\%20Sheet.pdf

Anzaldúa, Gloria (1987). Borderlands/La frontera. The New Mestiza. San Francisco: Aunt Lute.

Baez, Jesica (2014). La experiencia educativa trans. Los modos de vivir el cuerpo sexuado de los/as/xs jóvenes en la escuela secundaria de la Ciudad de Buenos Aires. Universidad de Buenos Aires (mineo) Tesis doctoral, Universidad de Buenos Aires, Argentina.

Belucci, Mabel y Rapisardi, Flavio (2001). Identidad: diversidad y desigualdad en las luchas políticas del presente. En: Teoría y filosofía política. La recuperación de los clásicos en el debate AAVV Buenos Aires CLACSO, Consejo Latinoamericano de Ciencias Sociales

Berkins (comp) (2007). Cumbia, copeteo y lágrimas. Buenos Aires. ALITT.

Butler, Judith (2010). Marcos de guerra. Las vidas lloradas. Buenos Aires: Paidós.

Cabral, Mauro (2007). La Paradoja Transgénero. En: www.ciudadaniasexual.org

Cabral, Mauro y Benzur, Gabriel (2005). Cuando digo intersex. Un diálogo introductorio a la intersexualidad. En: http://www.scielo.br/pdf/cpa/n24/n24a13.pdf

Fernández, Josefina (2004). Cuerpos desobedientes. Travestismo e identidad de género. Buenos Aires: Edit. Edhasa- IDAES

Foucault, Michel (2008). Historia de la sexualidad 1: la voluntad del saber. 2da. Edición. Buenos Aires: Siglo XXI Editores.

Haraway, Donna (1999). Las promesas de los monstruos: Una política regeneradora para otros inapropiables/bles En, Revista política y Sociedad, Nro.30, Madrid. 
INDEC (2012). Prueba Piloto, Primer Encuesta a la Poblacións Trans completo disponible en: http://www.indec.gov.ar/webencuestatrans/pp_encuesta_trans_set2012.pdf

Jones, Daniel; Libson, Micaela y Renata Hiller (2006). Sexualidades, política y violencia. La Marcha del Orgullo GLTTBI. Buenos Aires 2005. Segunda Encuesta Lugar: Buenos Aires.

Lopez Louro, Guacira (2010). L. Gênero, Sexualidade e Educação: uma perspectiva pósestruturalista. 11 ed. Petrópolis, RJ: Vozes, 2010.

Maffia, Diana (2001). Ciudadanía Sexual En Feminaria Año Nro. 26/27 Buenos Aires.

Mansilla, Gabriela (2015). Yo nena, yo princesa. Ed. Universidad Nacional Gral Sarmiento. Buenos Aires.

Morgade, Graciela (2011). Toda educación es sexual. Crujía Ediciones. Buenos Aires.

Parchuc, Juan Pablo y Lumbertno, María José (2008). Informe sobre códigos contravencionales y de faltas de las provincias de la República Argentina y la Ciudad Autónoma de Buenos Aires en relación con la discriminación y la represión a gays, lesbianas, bisexuales y trans. INADI.

Rockwell, Elsie (1996). La dinámica cultural en la escuela, En: Amelia Álvarez (edit.): Hacia un currículum cultural: la vigencia de Vygotski en la educación, Madrid, Fundación Infancia y Aprendizaje.

Sedgwick, Eve (1990). Epistemología del closet. Orientalismo Madrid: Ediciones Libertarias-Prodhufi.

Scott, Joan (2001). Experiencia, En: La ventana, № 13, México pp. 42-73.

Viola, Sabrina (2012). Autonomía progresiva de niños, niñas y adolescentes en el Código Civil: una deuda pendiente, En: Revista Cuestión de Derechos, Nro. 3. 